\title{
El empleo de «teams» en la docencia de teoría del derecho
}

\section{Marc-Abraham Puig Hernández}

Departamento de Ciencia Política y Derecho Público. Área de Filosofía del Derecho. Universidad Autónoma de Barcelona (UAB). Marcabraham.puig@uab.cat / https://orcid.org/0000-0002-1960-9903

\section{\$EWWDFW}

Face-to-face teaching in the 2020/2021 academic year has not always been possible. Hence, the Office 365 "TEAMS" app was used to complete the Legal Theory course at the Autonomous University of Barcelona (UAB), both in the lectures and in the seminar activities. In the latter case, we confine ourselves to a novel scenario because it requires argumentation at the moment, that is, spontaneous, and to be debated IQUKL overwhelmed by others. Therefore, resorting to a virtual medium did not presage satisfactory outcomes. However, the results contradict the initial perception: on the one hand, the evaluation had the same impact on the mark in the case of the seminars, but, on the other hand, the syllabus of philosophical content of the master classes, which is also linked to the argumentation, did not obtain the same performance due to losing those essential elements of the presence. In this work, an explanation of this phenomenon is offered.

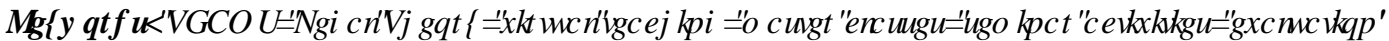

\section{HXP HQ}

La docencia presencial en el curso 2020/2021 no ha sido siempre posible. De ahí que se recurriera a la aplicación «TEAMS» de Office 365 para completar el curso de Teoría del Derecho en la Universidad Autónoma de Barcelona (UAB), tanto en las clases magistrales como en las actividades de seminario. En este último caso nos circunscribimos a un escenario novedoso debido a que en ellas se requiere de la argumentación en el momento, espontánea, y que se debata IQ UWX rodeados de personas críticas y expectantes. Por tanto, recurrir a un medio virtual no presagiaba resultados satisfactorios. Sin embargo, los resultados contradicen la percepción inicial: por una parte, la evaluación tuvo el mismo impacto sobre la nota en el caso de los seminarios, pero, por otra parte, el temario de contenido filosófico de las clases magistrales, vinculado igualmente con la argumentación, no obtuvo el mismo rendimiento al perder aquellos elementos imprescindibles de la presencialidad. En este trabajo se ofrece una explicación del fenómeno.

Palabras clave: TEAMS; Teoría del Derecho; docencia virtual; clases magistrales; actividades de seminario; evaluación

\section{Introducción}

La asignatura de Teoría del Derecho es una materia obligatoria de 6 créditos que se cursa durante el primer semestre del primer año de grado en la Facultad de Derecho y en la Facultad de Económicas de la Universidad Autónoma de Barcelona. La docencia se reparte en 4 horas semanales, de las cuales 
corresponden 2 horas por clase. Dependiendo del grado, el número de alumnos matriculados por grupo oscila entre los 100-130 en los de Derecho y unos 50 en los de ADE+Derecho.

A lo largo de esta asignatura se asientan las bases de un conocimiento genérico, universal, de lo que es el Derecho. Mientras que en el resto de disciplinas jurídicas el estudiante desarrolla y adquiere competencias desde un punto de vista científico, dado el objeto de estudio de estas disciplinas (por ejemplo, en Derecho Penal se estudia un objeto muy determinado, como lo es un código donde se regulan los delitos y las penas, y, además, las instituciones dogmáticas desarrolladas para esa labor, como el papel del denominado «dolo»), en Teoría del Derecho desenvuelve su capacidad de análisis teórico y universal sobre lo que es el ámbito jurídico, los conceptos jurídicos fundamentales, esto es, que no se circunscriben a una sola disciplina, sino a todas, y las cuestiones de técnica jurídica, como la interpretación y la aplicación del Derecho.

Por este motivo, la asignatura de Teoría del Derecho ubicada en el primer año del curso garantiza un conocimiento lo suficientemente genérico, esencial, de lo jurídico para ir enraizando el resto de saberes particulares y científicos que representa cada una del resto de las asignaturas de la carrera.

Ahora bien, en la Universidad Autónoma de Barcelona no se incluye en el último curso del grado la asignatura que vendría a ser la hermana gemela de Teoría del Derecho, a saber, la Filosofía del Derecho. A diferencia de los saberes científicos, al conocimiento teórico y universal del ámbito jurídico le acompaña una asignatura propia de la filosofía para evaluar, criticar, valorar el ordenamiento jurídico. Esta ausencia del plan docente de los estudios jurídicos nos obliga a dedicar la parte final de la docencia del curso de Teoría del Derecho a introducir algunos elementos clave de la reflexión filosófica, imprescindible para completar las competencias críticas y analíticas del alumnado, aquellas que no pueden desarrollarse únicamente desde un planteamiento cientificista de los asuntos humanos y de los problemas prácticos.

Esta serie de condicionantes, entre las que debemos contar que el conocimiento filosófico se realiza el primer año cuando preferiblemente debería acontecer en último curso al tener el alumnado un dominio de los saberes científicos parciales y una idea clara del estado general del ordenamiento jurídico, puede suponer una carga excesiva de lo cualitativo en unos estudios con los que se empieza a tomar contacto. Por otra parte, ello puede quedar compensado con el hecho de que los alumnos no arrastran asignaturas pendientes de otros años, sino que el primer curso siempre es una tabula rasa.

Teniendo en cuenta la gran diferencia del número de alumnos en diferentes grupos, se intenta equiparar la metodología de los grupos recurriendo a subgrupos reducidos de seminario para los del grado en Derecho (entre 40-50 alumnos). A las clases magistrales para todos los matriculados, vía online, se les acompaña de un trabajo autónomo del en el que deben entregar actividades quincenalmente en una sesión de seminario ya en el subgrupo al que pertenecen. En el curso académico 2020/2021 el 100\% de los alumnos efectúo este itinerario para la asignatura, sin que nadie llegara a notificar la opción de evaluación única.

La innovación viene de recurrir a Microsoft TEAMS para paliar la imposibilidad de llevar a cabo los seminarios virtuales, un recurso no previsto para el desarrollo de la docencia propia del ámbito filosófico. 
Para evaluar la experiencia recurriré a las evaluaciones, de ahí que los resultados a los que llegue deban ser catalogados de provisionales, quedando pendiente una valoración conjunta con el resto del área una vez finalizada la docencia anual del resto de nuestras asignaturas.

\section{Objetivos}

Debemos considerar que, entre los objetivos del aprendizaje del alumnado, figuran «Definir los principios básicos del ordenamiento jurídico» y asimismo «Demostrar un razonamiento reflexivo y crítico». Tareas propias de la Teoría del Derecho, por un lado, y de la Filosofía del Derecho, por otro. La idea que intento exponer es la de que en nuestra asignatura se imbrican dos mundos que aparentemente plantean una experiencia docente particular, característica y afanosa en preparar contenidos apropiados para el logro de tales objetivos. Al menos, porque los conocimientos teóricos siempre pueden reforzarse con la lectura de un buen manual, completo, puesto al día y de lectura más o menos amena pese al lenguaje técnico. Claro que, a ello, le sumamos una actividad propiamente filosófico-jurídica. Voy a intentar exponer con algo más de detalle este asunto, para evidenciar de qué manera nuestros objetivos quedan predeterminados.

La actividad filosófica aplicada al Derecho puede considerarse en una doble perspectiva: como una actividad propiamente dicha o como el resultado de la misma. Habiendo reservado una parte de la docencia para alcanzar el objetivo del plan docente, no es tan interesante, a fin de cuentas, el resultado de la actividad filosófica como la misma actividad. ¿Qué quiere eso decir? Pues que, si tenemos presente lo anteriormente dicho en la introducción, para adquirir las competencias iusfilosóficas con rigor debe cursarse toda una asignatura que prepare un contenido específico. Sin embargo, nos vemos limitados a tener que introducir el elemento más relevante de esa saber filosófico del Derecho. Y ese conocimiento priorizado no es otro que el hábito, la disposición, el uso continuo y habitual de la reflexión, la crítica, el análisis.

De esta manera, ya no es tanto adquirir una visión filosófica de todas las disciplinas jurídicas, sino, por el contrario, que los alumnos adquieran la competencia analítica y crítica propia de ese saber. Ya no es tanto plantearse y responder interrogantes de jaez utilitario, valorativo u ontológico; pasa por el método, la predisposición, el hábito. Digámoslo de un modo más rudimentario, no es tanto el qué de la disciplina filosófica, sino el cómo desenvolverse.

Los objetivos quedan predefinidos en dos: adquirir los conocimientos teóricos mínimos de lo que atañe a las disciplinas jurídicas y desenvolverse con cierta pericia en llevar a la práctica el análisis y la crítica de un objeto de estudio, en este caso, jurídico. A partir de ahí, la pregunta que nos urge responder es si cabe la posibilidad de aunar objetivos tan dispares en lo aparente, que requieren cada uno de ellos un trabajo específico, por no decir una asignatura propia en el grado.

\section{Desarrollo de la innovación}

Con la vista puesta al logro de tal fin, las clases teóricas online, a través de la plataforma TEAMS de Microsoft, se elaboraron partiendo de los manuales, como viene siendo habitual en cualquier clase fuera de medios virtuales. Como también viene siendo tónica frecuente, los alumnos suelen presentarse a la clase 
habiendo leído un mínimo contenido del temario que va a exponerse durante la sesión. Lo interesante de esta metodología es que el alumno dé un sentido coherente a todo lo que va leyendo y pueda comprender de dónde vienen los conceptos, por qué era necesario un conocimiento teórico y cuáles son los instrumentos que allí dispone para afrontar su profesión.

Claro que, hasta aquí, al lector poco o nada le habrá aportado o llamado la atención la entrada en escena de la aplicación TEAMS. A decir verdad, viene a ser exactamente lo mismo que una clase magistral en la facultad ahora trasladada al mundo virtual de las pantallas, las cámaras y los micrófonos, y por tanto a todas los beneficios e incomodidades que ello significa.

La novedad esencial viene de la mano de la adquisición de la competencia de las habilidades propias del saber filosófico. Y ello nos remite allende las clases magistrales, al trabajo autónomo no para esa clase, sino para la actividad de seminario y a la docencia respecto a los temas propios de la Filosofía del Derecho.

Respecto a la actividad de seminarios, en principio no estaba previsto recurrir a TEAMS para efectuarlas. Cuando dio comienzo el curso 2020/2021, estaba planeada la docencia magistral de manera virtual y la actividad seminarista presencialmente, a efectos de debatir y estimular la reflexión in situ de un tema preparado por cuenta propia previamente. Sin embargo, la imposibilidad de acudir a la facultad debido al confinamiento planteó un escenario en el que podía prescindirse de los seminarios, esto es, volcarlo todo en la docencia magistral, o por el contrario intentando llevar esa práctica al escenario online.

Para la consecución del segundo objetivo antes señalado no podía prescindirse del debate, de la reflexión compartida y del confrontamiento de opiniones. ¿Cómo puede desarrollarse una competencia práctica de tan relevante magnitud sin al menos poder interactuar? Ello llevó a plantear los seminarios a través del aplicativo TEAMS. Ahora bien, a diferencia de las clases magistrales, los seminarios no se grabaron.

Para aumentar la cantidad de material académico disponible para el alumnado, la docencia magistral virtual se graba y posteriormente se facilita haciéndola disponible para el grupo, por ejemplo, a través del campus virtual. Siempre ayuda recordar lo dicho en clase de un instrumento que ha venido a sustituir a los apuntes, como es un vídeo. Dejémoslo ahí, pues esta no es la cuestión ahora. Sin embargo, para estimular la participación en una actividad de carácter práctico, no teórico, se optó por no grabar las sesiones de seminario volcadas a la pantalla.

Es habitual que ante una clase grabada la asistencia virtual por parte del alumnado descienda considerablemente. Por mi experiencia esto no ha sido así y la asistencia siempre ha oscilado entre el 90 y el $100 \%$, siendo siempre justificados los casos de ausencia.

Respecto a los temas propios de la Filosofía del Derecho, se optó por acompañar la lectura de los manuales, por un lado, con textos filosóficos que analicen los aspectos vistos durante la semana y, por otro, con la elaboración de un pequeño escrito, no más de medio folio DIN-A4 en Word, con una pequeña crítica de la actividad preparada para el seminario. Esta reflexión en realidad debía partir de formarse una opinión 
propia sobre asuntos relevantes en el Derecho, por ejemplo, la cuestión de la obediencia a las normas, y justificarla según la lectura de los manuales y de los textos especializados. Una vez en el seminario, se hacía compartir la opinión de alumnos al azar y el resto debían criticarla.

\section{Resultados}

Una buena muestra de los resultados puede venir de la mano de las calificaciones.

La asignatura consta de dos tipos de prueba que configuran el 100\% total de la nota dividido en 50-50. La primera mitad corresponde a la actividad realizada en seminarios (asistencia y dos pruebas evaluables donde debía exponer su análisis y justificarlo según el trabajo realizado hasta el momento) y la segunda mitad al examen final.

La primera mitad de la nota refleja la actividad llevada a cabo por el alumno especialmente en los seminarios. Si bien en las primeras actividades y la primera prueba de evaluación aún renqueaba el hábito analítico y crítico (la puntación mayoría de las notas oscilaban entre el 6 y el 8), para la última prueba evaluable no hubo un solo alumno que no hubiese adquirido esa predisposición (subió la nota media hasta los márgenes del 8-10).

La adquisición de esa pauta crítica se asienta en la medida en que todos participan en el seminario y han podido contrastar sus opiniones fundamentadas en Derecho con las réplicas de sus compañeros.

En cambio, en el examen final la nota media volvió a bajar hasta el baremo inicial. Tengamos presente que en este tipo de examen el alumno debe sintetizar los conocimientos teóricos adquiridos de toda la asignatura y no desempeñar una labor crítica. En su mayor parte, su nota depende del estudio realizado por el alumno. Ahora bien, las preguntas que erraron en su respuesta fueron mayoritariamente las correspondientes a la parte del temario correspondiente a la Filosofía del Derecho, lo que contrasta con el buen trabajo realizado durante el curso.

\section{Conclusiones}

Los seminarios pudieron llevarse a cabo sin dificultades por vía TEAMS. Es cierto que en ocasiones las dificultades propias de la docencia online afectaron a los participantes, como los micrófonos o las cámaras, pero el aplicativo introduce el elemento escrito, por el cual, si bien cualquier dificultad como la conexión traba la participación espontánea del alumnado, compensa con su participación sintetizada en un chat.

La participación del alumnado cuenta con medios suficientes en TEAMS para poder llevarse a cabo. Cuando un alumno no puede participar por las circunstancias digitales del momento, se pasa al siguiente y el primero sabe que intervendrá por su cuenta o a petición del docente en otro momento. En realidad, algo similar sucede en los seminarios presenciales cuando algún alumno no se ha preparado bien el seminario y se salta al siguiente participante; sabe que otro día lo tiene que hacer mejor y que le tocará el turno en breve. 
Lo importante es que incluso con este tipo de dificultades el alumnado ha podido adquirir el hábito crítico fijado como objetivo a principios de curso. Lo que me interesa destacar es el por qué habiendo desarrollado esas competencias no se refleja en de unos pocos contenidos de esa disciplina filosófica.

De acuerdo con las tutorías y con las revisiones posteriores, los alumnos habían adquirido el hábito crítico para la práctica a través de los seminarios presenciales y virtuales, ahí no había diferencia destacable. Por el contrario, en cuanto al temario filosófico, al desarrollar la reflexión de forma telemática, las carencias en los medios para acceder a la docencia virtual con garantías mínimas les pasó factura.

Ese temario final correspondiente a la Filosofía del Derecho fue igualmente participativo. Por ejemplo, reflexionamos conjuntamente acerca del dilema moral de un tren que atropellará a una o a cinco personas según la dirección que tome. Quienes participaron en el debate el dilema no hizo más que reforzar el estudio de la materia que llevaban al día. Sin embargo, para aquellos con cualquier tipo de problemas digitales (conexión deficiente, sin micrófono, a través de un teléfono y con el ordenador para participar por escrito, etcétera) se evidenció la brecha.

La causa, el motivo, de esta brecha digital que tan bien se podía subsanar en la actividad de seminario, pero no en una sesión más conceptual, no ha sido otro que la pérdida del elemento humano en la educación. Porque el alumnado no podía participar como habitualmente venía haciéndolo y porque el docente no dispone del trato humano mínimo como atender visualmente a quienes están presentes en clase, identificando qué cuestiones cuestan más de comprender, qué alumnos andan perdidos en ese momento y pueden reconducirse, o qué cuestiones de un tema difícil generan más expectación y por tanto el resto del temario puede canalizarse por esa misma vía.

Digamos que, el empleo de TEAMS para la asignatura del Derecho tiene su parte buena y su parte mala. La buena es que la participación, la actividad clave metódica del alumnado, no se ha visto afectada en términos generales, pudiéndose corregir sobre la marcha esas pequeñas dificultades que siempre estarán ahí cuando recurramos al mundo digital. La mala es que perdemos la pericia humana, pero obviamente no aquella que nos sitúa tras una pantalla, sino lo humano de la educación que termina reflejándose objetivamente en la media al alza de un grupo, que es una cuestión añadida, cuantificable.

Digamos que, para solventar un curso académico tan convulso como el presente, TEAMS viene como anillo al dedo. Ahora bien, no podemos volcar la totalidad de la docencia a este medio, pues por el camino deshumanizamos no ya cualitativamente la educación superior, cuestión de por sí evidente, sino cuantitativamente. No obstante, recuerdo que la conclusión que comparte está condicionada por los resultados de la evaluación y la perspectiva provisional a expensas de cotejar los resultados con el resto de compañeros del área, pendiente de realizarse una vez finalizado el curso académico en su totalidad.

De ahí que TEAMS sea un instrumento que ha llegado para quedarse, especialmente para reuniones, tutorías y, en casos de fuerza mayor, para efectuar clases de seminario, participativas por parte del alumnado, sabiendo que el límite está en la transferencia de conocimiento, por parte del profesor al alumno, que necesita exponerse en persona. Es especialmente evidente en las disciplinas filosóficas, humanísticas, 
pues la brecha de este efecto con la parte teórica del temario, pese a darse igualmente, no era ni mucho menos tan pronunciada como en la parte filosófica del temario.

\section{Referencias}

KÖPCKE TINTURÉ, M. (2016). “Argumentar críticamente: Metodología docente y de evaluación para desarrollar esta competencia en la expresión escrita, oral y audiovisual” en Turull Rubinat, M. i Albertí Rovira, E. (eds.). QTIHSHUHQFDVGRFHQWALGHO* UDRIHQ' HHFKR. Barcelona: Octaedro- ICE. 